\title{
Pressure dependence of structural properties of ice VII: An ab initio molecular- dynamics study
}

Zdenek Futera, and Niall J. English

Citation: The Journal of Chemical Physics 148, 204505 (2018); doi: 10.1063/1.5022175

View online: https://doi.org/10.1063/1.5022175

View Table of Contents: http://aip.scitation.org/toc/jcp/148/20

Published by the American Institute of Physics

\section{Articles you may be interested in}

Proton dynamics and the phase diagram of dense water ice

The Journal of Chemical Physics 148, 214501 (2018); 10.1063/1.5028389

Communication: Diffusion constant in supercooled water as the Widom line is crossed in no man's land The Journal of Chemical Physics 148, 191102 (2018); 10.1063/1.5029822

Perspective: Ring-polymer instanton theory

The Journal of Chemical Physics 148, 200901 (2018); 10.1063/1.5028352

Theoretical prediction of crystallization kinetics of a supercooled Lennard-Jones fluid

The Journal of Chemical Physics 148, 204506 (2018); 10.1063/1.5021944

Aqueous solvation from the water perspective

The Journal of Chemical Physics 148, 234505 (2018); 10.1063/1.5034225

Announcement: Top reviewers for The Journal of Chemical Physics 2017

The Journal of Chemical Physics 149, 010201 (2018); 10.1063/1.5043197

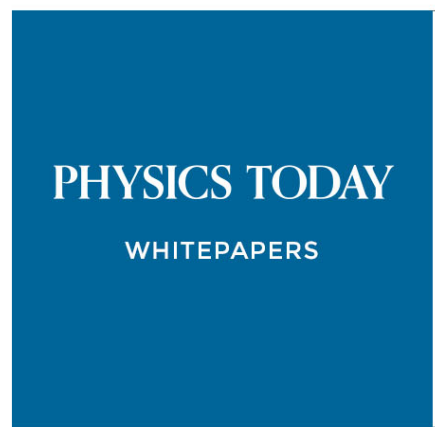

ADVANCED LIGHT CURE ADHESIVES

Take a closer look at what these environmentally friendly adhesive systems can do
READ NOW

PRESENTED BY

Q MASTERBOND 


\title{
Pressure dependence of structural properties of ice VII: An ab initio molecular-dynamics study
}

\author{
Zdenek Futera ${ }^{1,2, a)}$ and Niall J. English ${ }^{1, a)}$ \\ ${ }^{1}$ School of Chemical and Bioprocess Engineering, University College Dublin, Belfield, Dublin 4, Ireland \\ ${ }^{2}$ Department of Physics and Astronomy, University College London, Gower Street, London WC1E 6BT, \\ United Kingdom
}

(Received 11 January 2018; accepted 1 May 2018; published online 22 May 2018)

\begin{abstract}
The observed anomalous self-diffusivity of ice VII in the region of $10 \mathrm{GPa}$ at $\sim 400 \mathrm{~K}$ has been suggested to arise from a change in proton-hopping mechanism involving a transition from ionicdefect-driven diffusivity to that dominated by diffusion of rotational defects. Here, we report ab initio molecular dynamics to study the structural, hydrogen bonding, electronic, vibrational, and Raman properties of ice VII at this temperature and between 5 and $20 \mathrm{GPa}$ to elucidate any possible hints of intramolecular strain that may serve as precursor events for proton hopping to unfold. We determine such equilibrium properties to be in reasonable agreement with experimental Raman spectra, although we do not detect any water-dissociation and proton-hopping events per se, owing to still-large waterdissociation free-energy barriers. Published by AIP Publishing. https://doi.org/10.1063/1.5022175
\end{abstract}

\section{INTRODUCTION}

Of the 17 ice polymorphs determined experimentally so far, up to $200 \mathrm{GPa}$, ice VII possesses stability across a wideranging region above $2 \mathrm{GPa}^{1}$ Ice VII's structure consists of two inter-penetrating cubic-ice $\left(I_{\mathrm{c}}\right)$ sub-lattices, wherein protons locate at two of four equivalent crystallographic positions around oxygen atoms via Bernal-Fowler rules. ${ }^{2} I_{\mathrm{c}}$ sublattices are inter-connected with a disordered-proton motif via essentially random hydrogen bonding; however, the two inter-penetrating $I_{\mathrm{c}}$ sub-lattices are effectively independent, not being hydrogen-bonded together. Ice VII's simple structure offers an intriguing "playground" by which to dissect the quirks of proton quantal behavior, bobbing along the $\mathrm{O} \cdots \mathrm{O}$ double-minimum potential while under pressure. ${ }^{3,4}$ However, dramatic changes in ice VII properties have been witnessed at relatively low pressures $(\sim 10-15 \mathrm{GPa})$ where quantum effects are mild. Room-temperature X-ray-diffraction-peak structures, most pronounced at $\sim 13 \mathrm{GPa}$ in their splitting, are suggestive of partial proton ordering mediating reductions in crystal symmetry and increases in ferroelastic character, ${ }^{5-7}$ supported by high-pressure Raman spectroscopy. ${ }^{7-9}$ However, neutron powder-diffraction measurements do not reveal diffraction-peak splitting ${ }^{3,10}$ but suggest interstitial-proton appearance at $\sim 13 \mathrm{GPa}$ in deuterated ice VII, although inelastic X-ray scattering does not support this interstitial-proton picture. ${ }^{11}$ Ice VII ab initio molecular dynamics (AIMD) simulation showed essentially no water intramolecular dissociation up to $40 \mathrm{GPa}$ and $800 \mathrm{~K}^{.11}$

Intriguingly, Okada et al. reported anomalous protonconductivity results in the 273-303 $\mathrm{K}$ range at around

\footnotetext{
a) Authors to whom correspondence should be addressed: z.futera@ucl.ac.uk and niall.english@ucd.ie, Tel.: +353-1-7161646, Fax: +353-1-7161177.
}

$10 \mathrm{GPa}$, with minimal activation energy thereat. ${ }^{12}$ Investigating proton-diffusivity pressure dependence, Katoh et al. measured these at $\sim 10,30$, and $60 \mathrm{GPa}$ and $400 \mathrm{~K}$ via diamondanvil cell (DAC) and IR-reflection spectroscopy, ${ }^{13}$ finding exponentially declining proton conductivity with increasing pressure, although the pressure spacing of data around $10 \mathrm{GPa}$ was sparse. Noguchi and Okuchi applied microRaman spectroscopy and DAC techniques to determine proton (and oxygen) diffusivity in the 400-425 $\mathrm{K}$ and 5.5-17 GPa ranges, finding a substantial maximum at $\sim 10 \mathrm{GPa},{ }^{14}$ indicating that protonic diffusion dominates ice VII's hydrogen diffusion-in stark contrast to the ice I $h$ 's whole-molecule interstitial mechanism. It was suggested that the $\sim 10 \mathrm{GPa}$ anomaly arises from a change in proton-diffusion mechanism from ionic-defect diffusion to that of rotational defects as the rate-determining steps, ${ }^{12}$ not inconsistent with Refs. 13 and 14 .

In any event, bearing in mind some evidence for protonic diffusivity in ice VII and changeover in proton-diffusivity mechanism maximising this at $\sim 10 \mathrm{GPa},{ }^{12-14}$ a detailed elucidation of ice VII's structural, hydrogen bonding, vibrational, and Raman properties, particularly in the region of $\sim 10 \mathrm{GPa}$, would be of substantial benefit to glean a more complete understanding of its underlying physical properties potentially facilitating this proposed transition in proton diffusivity. In particular, scrutiny of Raman, IR-spectrum, and proton density of states (DOS) would potentially shed light on underlying proton dynamical behavior. In the present study, motivated by these open questions, we perform high-quality ab initio molecular dynamics (AIMD), featuring state-of-theart non-local treatment of dispersion, to probe, inter alia, these properties at 5-20 GPa; in so doing, we determine such properties to be in reasonable accord with experimental Raman spectra, although we do not detect direct evidence of water-dissociation and proton-hopping events per se, 
as recorded in some studies (e.g., Ref. 14), owing to large water-dissociation free-energy barriers. Important new insights relate to the influence of pressure compaction on structural and spectral response in ice VII, in that relatively closer interaction of the quasi-independent, inter-penetrating $I_{\mathrm{c}}$ sublattices at high pressures leads to spectral shifts in librational and stretch motions, as well as a likelihood of enhanced intermolecular electron-cloud overlap that leads to the development of a dramatic lower frequency shoulder - a telltale "signature" of more strongly interacting and penetrating electron clouds.

\section{METHODOLOGY}

$A b$ initio molecular dynamics (AIMD) simulations were done within the framework of Density Functional Theory (DFT) in generalised-gradient approximation (GGA). We employed the van der Waals-density (vdW-DF) exchangecorrelation functional with an explicit non-local DionRydberg-Schroder-Langreth-Lundqvist (DRSLL) correlation correction to involve van der Waals interactions. ${ }^{15,16}$ This functional was recently shown to perform exceptionally well for liquid water, ice, and clathrate hydrates. ${ }^{17-24}$ Secondgeneration Car-Parrinello MD MD,25,26 $^{2}$ was carried out with a 1 fs time step in the canonical (NVT) ensemble after careful equilibration of the system. The system temperature was set at a target of $410 \mathrm{~K}$ by a Langevin thermostat, with the $\gamma$ factor set to 0.005 . The calculations were performed using the TZV2P basis set and Goedecker-Teter-Hutter (GTH) pseudopotentials $^{27}$ using the CP2K software package. ${ }^{28}$ A cubic $2 \times 2 \times 2$ supercell of the 16-molecule ice VII unit cell was studied, consisting of 128 water molecules. ${ }^{4,17,18}$ The lattice constants of the cubic system (see Table I) were calibrated carefully to yield pressures of 5, 10, 15, and $20 \mathrm{GPa}$ for MD at $410 \mathrm{~K}$, with subsequent $0.1 \mathrm{~ns}$ of "production" dynamics at each pressure.

To analyze the structural properties of adsorbed water, we calculated site-site radial distribution functions (RDFs), as well as water $\mathrm{O}_{\mathrm{w}}-\mathrm{H}_{\mathrm{w}}$ bond lengths, $\mathrm{H}_{\mathrm{w}}-\mathrm{O}_{\mathrm{w}}-\mathrm{H}_{\mathrm{w}}$ valence angles, and individual-water dipoles as mean values $\langle x\rangle=\int x P(x) d x$ of the corresponding normalised histograms $P(x)$ collected from the simulations. Similarly, histograms of hydrogen-bond A-H lengths and A-D-H angles were computated. The hydrogen bonds were selected on the basis of geometry criteria for the arrangement of donor (D), acceptor (A), and the hydrogen: the maximum allowed A-D distance was $3.5 \AA$, and the A-D-H angle could not exceed $35^{\circ}$.

TABLE I. Structural parameters of ice VII collected from the $a b$ initio MD simulations performed at $410 \mathrm{~K}$ under different pressures $p(\mathrm{GPa})$ : cell size $L$ ( $)$, lattice constant $a(\AA)$, shortest $\mathrm{O}-\mathrm{O}$ distance $r_{\mathrm{OO}}(\AA), \mathrm{O}-\mathrm{H}$ bond length $r_{\mathrm{OH}}(\AA), \mathrm{H}-\mathrm{O}-\mathrm{H}$ valence angle $\alpha_{\mathrm{HOH}}(\mathrm{deg})$, atomic Hirshfeld charge on oxygen $q_{\mathrm{O}}$, molecular dipole moment $\mu$ (D).

\begin{tabular}{lccccccc}
\hline \hline$p$ & $L$ & $a$ & $r_{\mathrm{OO}}$ & $r_{\mathrm{OH}}$ & $\alpha_{\mathrm{HOH}}$ & $q_{\mathrm{O}}$ & $\mu$ \\
\hline 5 & 12.924 & 3.231 & 2.798 & 1.000 & 105.005 & -0.503 & 1.473 \\
10 & 12.533 & 3.133 & 2.714 & 1.005 & 105.265 & -0.499 & 1.464 \\
15 & 12.265 & 3.066 & 2.655 & 1.009 & 105.485 & -0.496 & 1.457 \\
20 & 12.057 & 3.014 & 2.610 & 1.013 & 105.686 & -0.493 & 1.450 \\
\hline \hline
\end{tabular}

The IR spectrum was computed as the power spectrum of the system-collective-dipole auto-correlation function

$$
I(\omega) \propto \omega^{2} \int\left\langle\boldsymbol{M}\left(t_{0}\right) \boldsymbol{M}\left(t_{0}+t\right)\right\rangle e^{-i \omega t} d t,
$$

where $\boldsymbol{M}(t)$ can be written as the sum of individual water dipoles $\boldsymbol{M}(t)=\sum_{i} \boldsymbol{\mu}(t)$. The vibrational density of states (VDOS) was calculated by Fourier transforming the respective velocity-autocorrelation function accumulated from 0.1-nslong trajectories,

$$
I(\omega) \propto \int\left\langle\boldsymbol{v}_{H}\left(t_{0}\right) \cdot \boldsymbol{v}_{H}\left(t_{0}+t\right)\right\rangle e^{-i \omega t} d t
$$

The auto-correlation function itself was computed on the basis of Wiener-Khintchine relations, ${ }^{29,30}$ employing the fast Fourier transform (FFT) algorithm with efficient $O(n \log n)$ scaling,

$$
\left\langle f\left(t_{0}\right) f\left(t_{0}+t\right)\right\rangle=\frac{1}{2 \pi} \int\left|\int f\left(t^{\prime}\right) e^{-i \omega t^{\prime}} d t^{\prime}\right|^{2} e^{i \omega t} d \omega .
$$

To obtain vibrational Raman spectra, we Fouriertransformed the autocorrelation function of hydrogen velocities projected onto the $\mathrm{O}-\mathrm{H}$ bond vector. In this way, the vibrational spectrum is decoupled from the translational and rotational modes and is therefore directly comparable with experimentally measured data.

\section{RESULTS AND DISCUSSION}

To assess structural changes in ice VII under different pressures, we first calculated the radial distribution function (RDF) for O-O pair interactions-cf. Fig. 1. At first glance, it is obvious that there is substantial shortening of intermolecular distances in the crystal as the pressure increases from 5 to $20 \mathrm{GPa}$. This is, of course, expected because the system is compressed by external pressure. The position of the first sharp RDF peak corresponds to the shortest $\mathrm{O}-\mathrm{O}$ inter-molecular distance $\boldsymbol{r}_{\mathbf{O O O}}$, which is related to the lattice constant, $\boldsymbol{a}$, by $\boldsymbol{r}_{\text {OO }}=\boldsymbol{a} \sqrt{\mathbf{3}} / \mathbf{2}$ in a perfect ice VII lattice. The values obtained from the simulations, collected in Table I, are in essentially exact agreement with this relationship. The ice VII lattice is therefore well preserved structurally in 0.1 ns_long AIMD in 5-20 GPa pressure range. The second

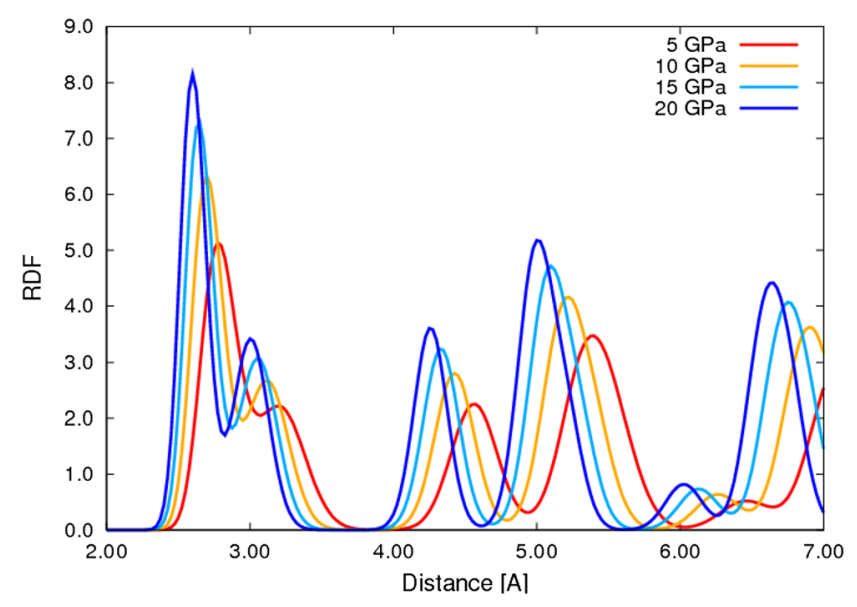

FIG. 1. Radial distribution function (RDF) for $\mathrm{O}-\mathrm{O}$ pair interactions under pressures of $5,10,15$, and $20 \mathrm{GPa}$. 
RDF peak, which, together with the first one, determines the nearest neighbour shell of each water molecule, is the shortest distance between water molecules from the two ice VII sublattices. This peak position determines directly the unit-cell lattice constant. Their numerical values at different pressures can be found in Table I. Similarly, the wider two-peak band between 4 and $6 \AA$ indicates the second hydration shell, and so on for subsequent RDFs and progressively more distant outer shells.

The compression of the ice VII crystal not only causes the expected inter-molecular distance shortening, as described above but also affects the (intramolecular) structure of the water molecules themselves. This can be seen in the calculated distribution of the $\mathrm{O}-\mathrm{H}$ bond lengths and $\mathrm{H}-\mathrm{O}-\mathrm{H}$ angles, which are shown in Fig. S1 of the supplementary material. The mean values of these distributions are listed in Table I. O-H bonds are elongated slightly from $1.000 \AA$ at a pressure of $5 \mathrm{GPa}$ to $1.013 \AA$ at $20 \mathrm{GPa}$, which has been remarked before. ${ }^{31}$ Simultaneously, the intramolecular water angle increases from $105.005^{\circ}$ to $105.686^{\circ}$ in going from 5 to $20 \mathrm{GPa}$, respectively. Note that a mean bond length of $0.993 \AA$ and angle of $105.335^{\circ}$ were found for liquid water under ambient conditions using the same DFT. ${ }^{23}$

Obviously, as the molecules become closer under higher pressure, it can be conjectured not unreasonably that their charge densities become somewhat more overlapped and the hydrogen atom is interacting not only with the oxygen in the same molecule but partly also with oxygen lone pairs on the nearest neighbours, which participate in hydrogen bonding. This effect can be seen on the charge-density isosurface that we calculated on snapshots from MD simulations under $5 \mathrm{GPa}$ and $20 \mathrm{GPa}$ pressure (cf. Fig. 2). Although the charge density is localised only on individual water molecules in the $5 \mathrm{GPa}$ structure (considering an isovalue of 0.05 a.u.), it overlaps clearly in the structure under $20 \mathrm{GPa}$ pressure. To show the density changes in the intermolecular region more quantitatively, we calculated the density profile on the $\mathrm{O}-\mathrm{O}$ vector averaged over all the nearest neighbours in the structure. The profile can be found in the supplementary material (cf. Fig. S2) and was normalised for better comparison of systems with different lattice constants. Charge density decays rapidly with distance from the oxygen centre; however, its increase in the inter-molecular region as the pressure rises is observable clearly. This partial sharing, or delocalisation, of the hydrogen may be considered logically to lead to some degree of $\mathrm{O}-\mathrm{H}$ bond elongation, as is seen upon increasing pressure (cf. Table I). Indeed, each of the respective standard deviations in the $\mathrm{O}-\mathrm{H}$ bond length for 5-20 GPa was $\sim 3.5 \times 10^{-3}$; pairwise $t$-tests between the bond lengths at each pressure indicate that the null hypothesis $\left(H_{0}\right)$ is only rejected beyond $90 \%$ certainty for 5 vs $20 \mathrm{GPa}$. This renders this delocalisation argument necessarily tentative and reinforces it as fundamentally conjectural in nature. Although here we did not calculate the free-energy barrier for proton transfer between neighbouring water molecules, one may expect that this barrier becomes lowered with higher pressure.

Charge redistribution described above is visible also upon gauging changes in Hirshfeld atomic charges. Their
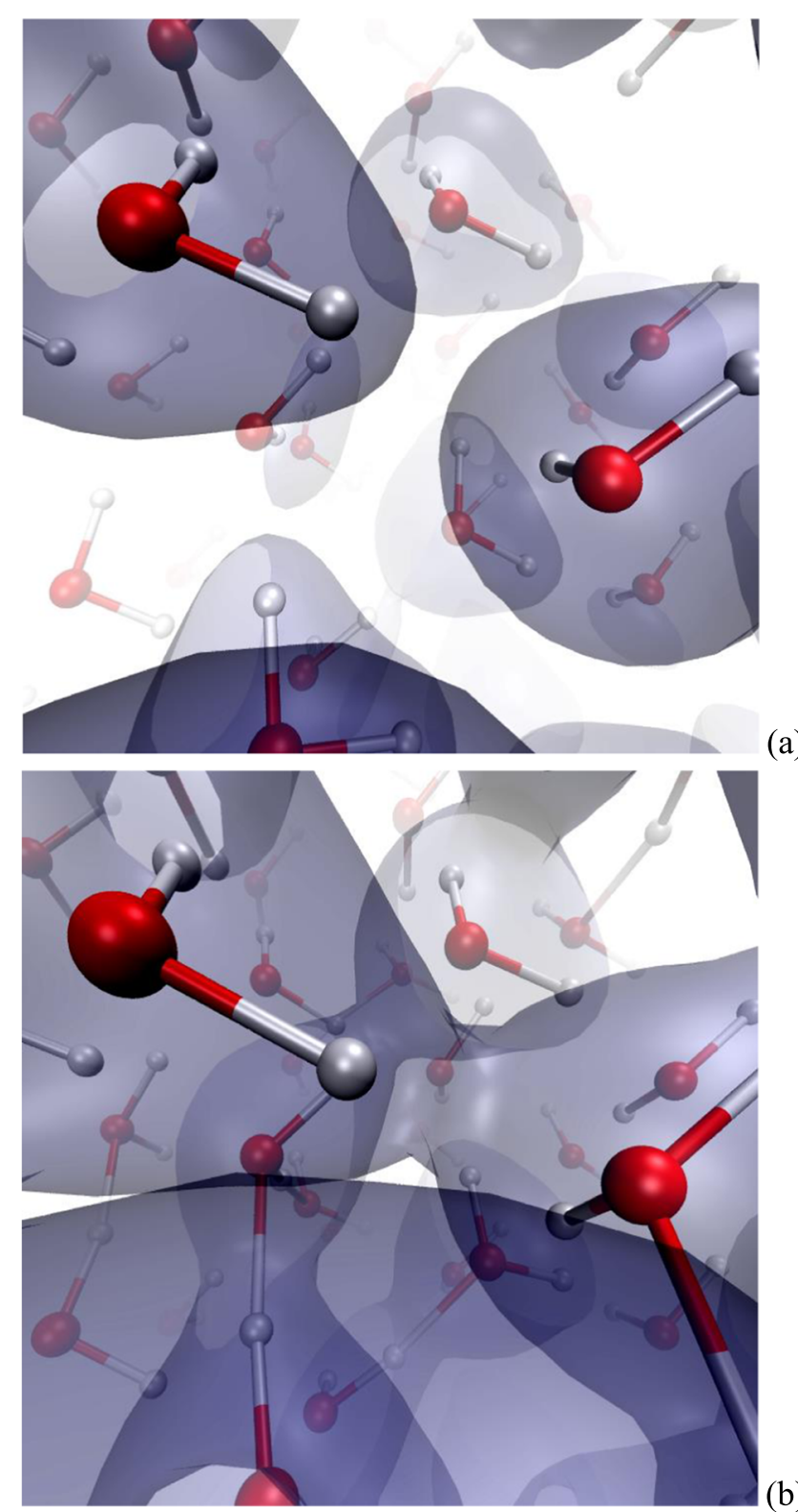

(b)

FIG. 2. Snapshot of the structure and charge density (plotted as a surface at 0.05 a.u. isovalue) of ice VII under (a) $5 \mathrm{GPa}$ and (b) $20 \mathrm{GPa}$ pressure.

distribution collected from the AIMD trajectories is shown in Fig. S3(a) of the supplementary material, while the mean value of charge on oxygen atoms is listed in Table I. As the molecules are neutral, the hydrogen charge is simply half the oxygen one but with the opposite sign. The oxygen atom becomes less negative as the pressure is increased, changing from -0.503 to $-0.493 e$ on going from 5 to $20 \mathrm{GPa}$, respectively (compared with $-0.506 e$ found in liquid water under ambient conditions with the same functional). Although the $\mathrm{O}-\mathrm{H}$ bond lengths become elongated at higher pressure, the wider molecular angles and reduced charge differences cause a decrease in the molecular dipole moment, which is reduced from 1.473 to $1.450 \mathrm{D}$ upon increasing pressure from 5 to $20 \mathrm{GPa}$ [cf. distributions in Fig. S3(b) of the supplementary material and also their mean values in Table I]. The molecular dipole moment in ice VII under pressure of $10 \mathrm{GPa}$ is practically identical to that in the ambient-condition liquid state 
(1.465 D) found in Ref. 23, although, naturally, the molecular structure is markedly different.

Regarding the charge-density redistribution discussed above, one can expect also concomitant changes in electronic levels of the system. In Fig. 3, we depict the projected density of states of ice VII in the valence-band, bandgap, and conductionband edge regions, as they were calculated on MD snapshots from simulations under different pressures. At first glance, it can be seen that occupied bands remain intact. The valenceband edge is formed by oxygen $\pi$-orbitals, hybridised partly with hydrogen $s$-states. Indeed, this structure is typical for all condensed-phase water systems. Nevertheless, it is obvious that pressurising ice VII causes wider opening of the bandgap. This is caused by the repulsion of $\sigma^{*}$ anti-bonding orbitals formed by hydrogen functions and located further from the oxygen nuclear centre. The numerical value of the bandgap is of course underestimated in our calculations because of a wellknown self-interaction error present in the GGA functionals (see Fig. S4 of the supplementary material, where the effect of exact exchange involved in hybrid functions is shown); however, the bandgap opening with increasing pressure is a physical effect.

Naturally, pressure-induced structural compression of the ice VII crystal can be seen also in hydrogen bonding between water molecules. Each water molecule is hydrogen-bonded to its four neighbours from the same sub-lattice, while the two sub-lattices are not connected by hydrogen bonds. We applied the geometric-identification criteria described above without observing any hydrogen-bond breaking/formation during $0.1 \mathrm{~ns}$ AIMD; this indicates that molecules are essentially vibrating along their equilibrium positions, with little appreciable rotation. The changes in hydrogen-bond lengths and angles can be seen in Fig. 4's distributions and their mean values (cf. Table II). As expected, hydrogen-bond lengths are shorter, with lower amplitude fluctuations therein, at higher pressure: here, the proton is interacting more strongly with the oxygen from the neighbouring molecule (i.e., the

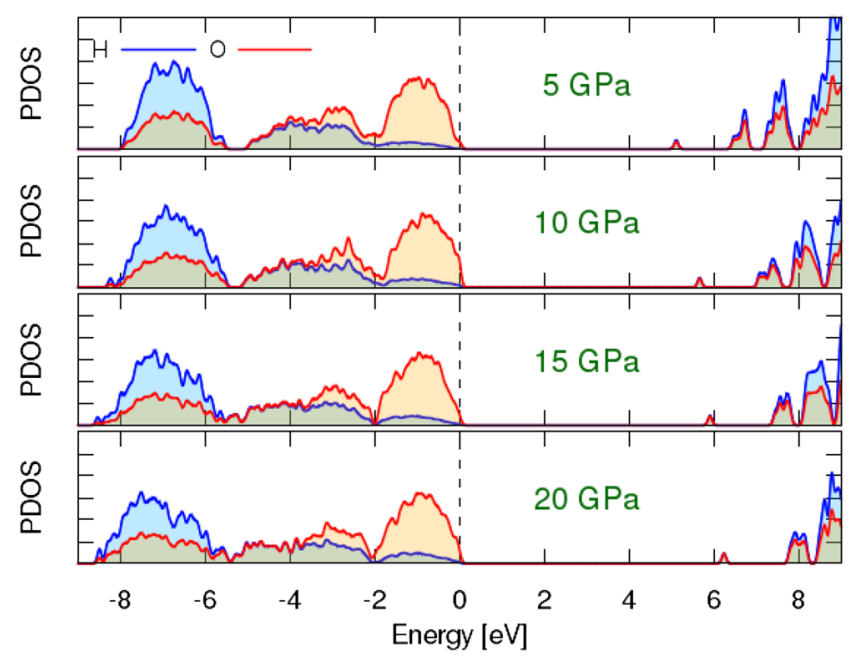

FIG. 3. Effect of pressure on electronic states of ice VII. Projected density of states (PDOS) of hydrogen (blue) and oxygen (red) is shown. The states are aligned to the valence-band edge, which is used as a reference. The occupied states are therefore negative, while empty states are in the positive region of the plot.
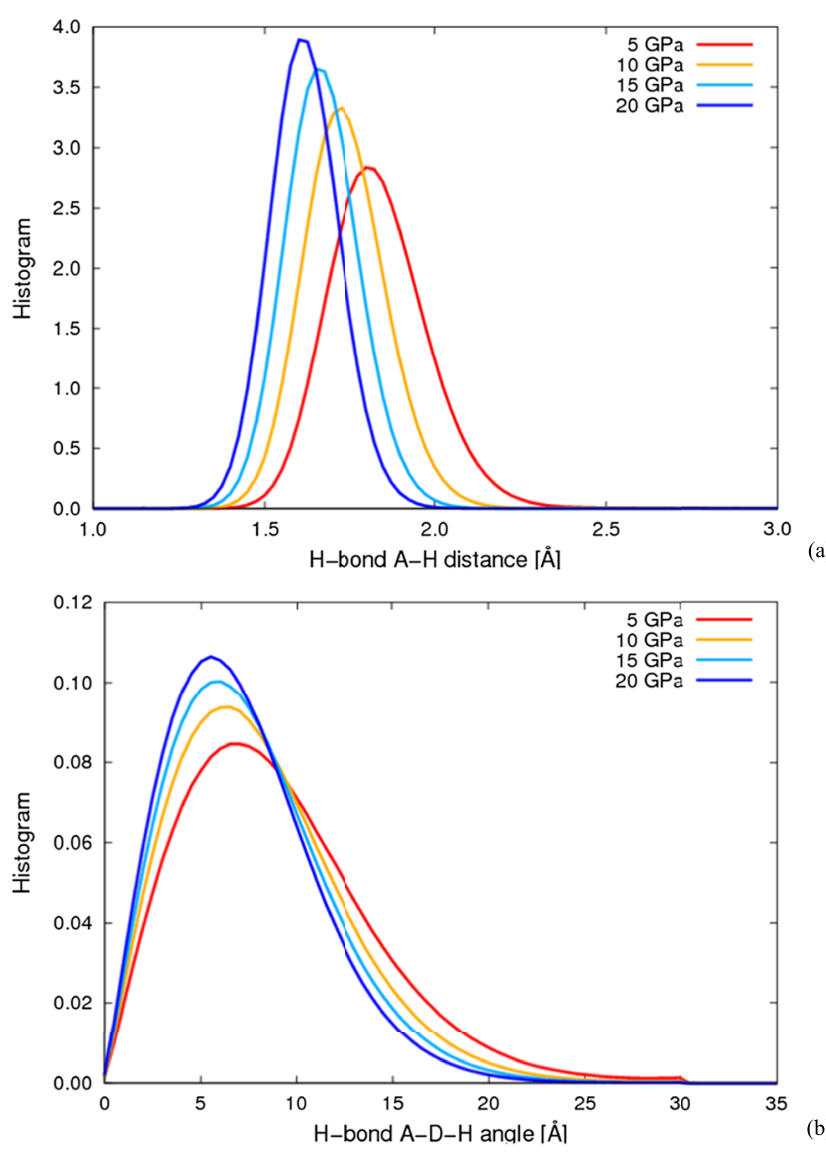

FIG. 4. Distributions of hydrogen-bond (a) A-H distance and (b) A-D-H angle under pressures of 5, 10, 15, and $20 \mathrm{GPa}$.

hydrogen-bond acceptor). This shortening upon greater pressurisation is illustrated starkly from $1.834 \AA$ at $5 \mathrm{GPa}$ (compared with $1.947 \AA$ in ambient-condition liquid water) to $1.620 \AA$ at $20 \mathrm{GPa}$; in essence, the hydrogen is forced to align better with a line connecting the oxygen atoms (the hydrogenbond donors and acceptors), thus reducing the A-D-H angle at higher pressure. This effect was also seen in the opening of the intra-molecular angle, discussed above, although the pressure-induced inter-molecular hydrogen-bond effect is the underlying mechanistic cause.

Energetically, the interaction between molecules in the crystal state can be quantified as the difference between the interaction or formation energy of a molecule in the crystal and in vacuum, i.e., $E_{\text {int }}=E_{\text {crystal }} / N_{m o l}-E_{m o l}^{v a c}$. The interaction energy involves not only inter-molecular electrostatic and van der Waals interactions but also a deformation contribution, which is the energy needed to bring a water molecule

TABLE II. Hydrogen-bond length $d_{\mathrm{DH}}(\AA)$, hydrogen-bond angle $\alpha_{\mathrm{ADH}}$ (deg), interaction energy $E_{\text {int }}(\mathrm{kcal} / \mathrm{mol})$, and water-molecule deformation energy $E_{\text {def }}(\mathrm{kcal} / \mathrm{mol})$.

\begin{tabular}{lcccc}
\hline \hline$p$ & $d_{\mathrm{DH}}$ & $\alpha_{\mathrm{ADH}}$ & $E_{\text {int }}$ & $E_{\text {def }}$ \\
\hline 5 & 1.834 & 9.040 & -12.584 & 0.726 \\
10 & 1.737 & 8.121 & -10.848 & 1.464 \\
15 & 1.671 & 7.574 & -9.476 & 1.486 \\
20 & 1.620 & 7.174 & -7.936 & 1.591 \\
\hline \hline
\end{tabular}


in vacuum from its optimal gas-phase structure to the structure that it has in the crystal (i.e., $E_{d e f}=E_{\text {mol }}^{\text {crys }}-E_{\text {mol }}^{\text {vac }}$, or the difference in gas-phase self-energies of the molecules in their respective crystal- and vacuum-optimised configurations; note that in contrast to the formation energy, the deformation contribution is single-molecule property). Both interaction energies and their deformation contributions for each considered pressure are listed in Table II. The interaction energy becomes less negative as the pressure increases. This is caused partly by an increase in deformation energy as the water bonds become more elongated and the intramolecular angle becomes larger, and also by enhanced inter-molecular repulsion at short distances, which becomes more important as the system is compressed at higher pressures.

It is instructive to investigate water-molecule vibrations and their response to changes in pressure. First, we calculated vibrational density of states (VDOS) by Fourier transforming the velocity autocorrelation function (vide supra). Indeed, computational VDOS might have more vibrational peaks than experimentally measured IR spectra where some of them could be inactive because of selection-rule restrictions. Therefore, we also Fourier-transformed the dipole autocorrelation function to obtain the IR spectra directly. These two approaches are compared in Fig. 5. All three bands typical for water systemslibration, bending, and stretching — can be seen in the spectra, and the two approaches (VDOS vs. IR) differ only in the intensity of the peaks due to presence of low-frequency libration
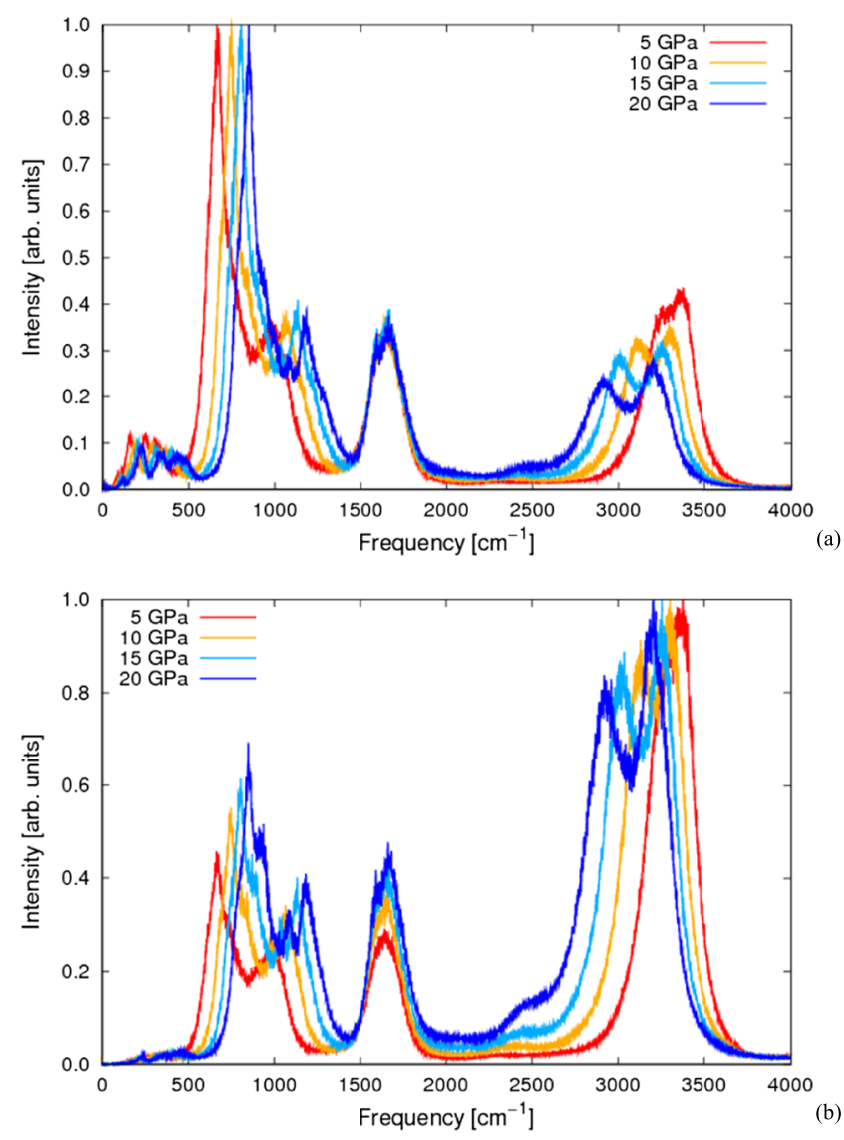

FIG. 5. (a) VDOS calculated from velocity autocorrelation function and (b) IR spectra obtained from dipole autocorrelation function. Both spectra were calculated for pressures of 5,10,15, and $20 \mathrm{GPa}$. modes. Compression of the system by application of external pressure leads to red-shifting of the librational band and, simultaneously, blue-shifting of the stretching band, while the bending modes remain largely unaltered. Dispersion of the stretching band to its symmetric and anti-symmetric contributions is more pronounced at higher pressure; at $5 \mathrm{GPa}$, only one broad stretching peak like in liquid water is visible.

Because the water molecules are organised in a crystal lattice, the spectra exhibit significant directional dispersion, as can be seen in Figs. S5 and S6 of the supplementary material. This is especially the case of the IR spectra calculated from the dipole autocorrelation function. The libration band is visible not only in the $y$-and $z$-components of the spectrum but also in the $x$-direction. On the other hand, the bending modes are contributing only in the $x$-component of the spectra. However, the crystal structure of ice VII (taken to the limit of infinite size) consists of random hydrogen bonds, which would therefore be isotropic in this limit. Therefore, this anisotropy appears to be an artefact of the necessarily small systems (in an absolute sense) with which we must work with computationally demanding state-of-the-art DFT. In the limit of an infinite cell, the random hydrogen-bond arrangement would serve to eliminate any anisotropy.

To obtain a closer look on changes in the stretching band, we extracted these vibrations by calculation autocorrelation function of hydrogen velocities projected on the $\mathrm{O}-\mathrm{H}$ bond vector and its subsequent Fourier transform. This spectrum, which is a good approximation to vibrational Raman measurements, is shown in Fig. 6. The stretching band is located between 3000 and $3500 \mathrm{~cm}^{-1}$ at a relatively low pressure of $5 \mathrm{GPa}$, which is more narrow and partly blue-shifted compared with liquid water under ambient conditions where this band can be found between 3000 and $3900 \mathrm{~cm}^{-1}$. As soon as the pressure increases to $10 \mathrm{GPa}$, the dispersion to anti/symmetric modes is visible. At the higher $20 \mathrm{GPa}$ pressure, a low-frequency shoulder of the band appears near $2500 \mathrm{~cm}^{-1}$.

In terms of physical insights from the vibrational and Raman spectra, the lattice compression at higher pressures leads to a greater degree of inter-connection of the $I_{\mathrm{c}}$

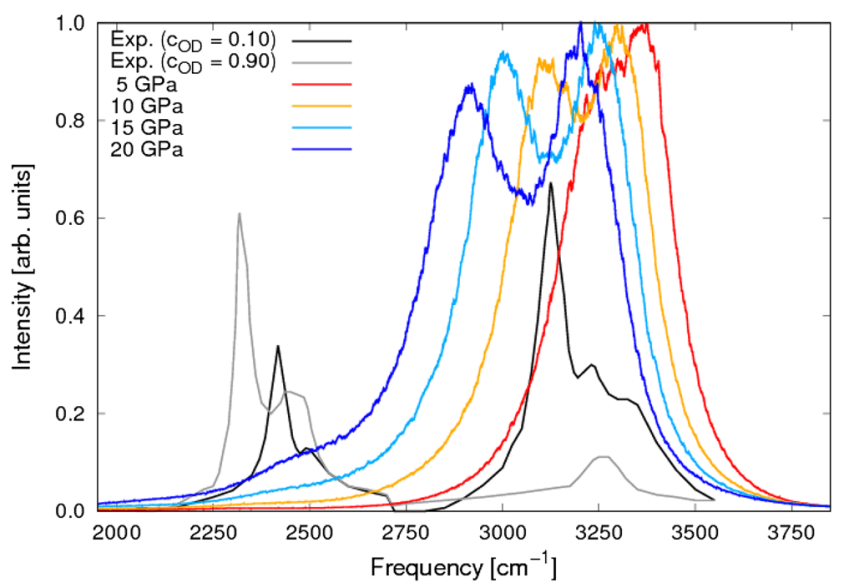

FIG. 6. Stretching band of the Raman vibrational spectrum, calculated from the autocorrelation function of hydrogen velocities projected to the $\mathrm{O}-\mathrm{H}$ bond vector. Experimental curves from Ref. 14 measured at 8.1 GPa are shown for comparison, where $c_{\mathrm{OD}}$ indicates the molar deuterium concentration $[\mathrm{D} /(\mathrm{H}+\mathrm{D})]$. 
sub-lattices via the disordered-proton motif vis-à-vis lower pressures (albeit still having a good degree of de-facto independence in absolute terms even at $20 \mathrm{GPa}$ ). This helps to explain the respective red- and blue shifting of the librational and stretching bands, in that increasing pressure compaction and closer sub-lattices tend to reduce the oscillations in rotational motion (i.e., librations) away from a less hindered, free-rotor state while allowing leakage of intramolecular vibrational energy into $\mathrm{OH}$-stretch modes to fluctuate more rapidly (at higher frequency). More tentatively, a greater level of inter-molecular electron-cloud overlap (vide supra) upon increasing levels of compression serves to promote the mounting dispersion to anti/symmetric modes observed in the Raman spectra, especially with the rather noteworthy appearance of the low-frequency shoulder near $2500 \mathrm{~cm}^{-1}$; as in the case of closer inter-connection of the $I_{\mathrm{c}}$ sub-lattices (in relative terms) leading to lower librational frequencies due to greater levels of "communication" and interaction, the enhanced inter-molecular electron-cloud overlap (justified partly by the increasing intra-molecular $\mathrm{OH}$ distance) leads to the development of a lower frequency Raman-stretch shoulder.

Detailed experimental Raman spectra are reported in Fig. 6 of Ref. 14 as a function of the level of deuteration at a similar temperature and pressure range, as studied here. For easier comparison, we show these experimental curves also in Fig. 6, together with the calculated data. For low levels of deuteration [i.e., $\mathrm{D} /(\mathrm{H}+\mathrm{D})=10 \%$ ], we see the H-related peak at $\sim 3300 \mathrm{~cm}^{-1}$ in the present work in semi-quantitative agreement with that for the low-deuterated case experimentally (albeit centred at $\sim 3100 \mathrm{~cm}^{-1}$ ), with a similar shape and shoulder structure. The higher Raman-frequency-mode values for AIMD with respect to the experiment are most likely a consequence of classical molecular dynamics rather than the quantal motion; ${ }^{24}$ so this level of agreement for the H-related Raman mode is satisfactory. However, in terms of accord with the experiment more generally, the lack of observation of proton hopping in the present work belies such observations of proton diffusivity in Ref. 14, and this arises most probably out of insurmountable energy barriers for such hopping events in the present case as regards standard AI-MD (i.e., without any biasing). Aside from Raman and proton-hopping data, there is not a great deal of further experimental data available for direct comparison with experiment; in part, this prompted us to study ice VII using some of the most sophisticated and accessible electronic-structure methods in the present study.

An estimation of quantum effects in altering the protonhopping (free-energy) energy barrier would require calculation of the proton-transfer energy barrier along plausible oxygento-oxygen nearest-neighbour-to-neighbour hopping pathways. However, such an estimation, even in the classical-protonicmotion approximation via, for instance, umbrella sampling (US), would be computationally prohibitive with any level of rigour under periodic boundary conditions (PBCs) in the full supercell using the vdW-DF treatment, as used in the present study. Naturally, the accurate path-integral (PI) treatment of the same phenomenon via PI-adjusted umbrella sampling $32-34$ would be, ipso facto, even more prohibitive (e.g., of the general order of 16-32 times so, depending on the number of quantum beads needed for a converged estimate, etc.). However, it is nonetheless interesting to speculate on how nuclear quantum effects (NQEs) in proton motion would be expected to affect these energy barriers. In particular, in the very interesting recent work, Rossi et al. have estimated NQE on proton self-diffusivity in explicitly protonated water wires (containing only $\sim 20$ water molecules) via (thermostatted) ring-polymer (RP-) MD using dispersion-adjusted DFT ${ }^{35}$ although this does not generate an explicit free-energy barrier per se, unlike PIadjusted umbrella sampling in Refs. 32-34, it does afford an excellent insight into competing quantal-versus classicalmotion effects for protons. Here, it was found that NQE, accounted for in the guise of RP-MD, served to increase proton-hopping self-diffusivity for BLYP+vdW treatment by $\sim 5 \%$ to $48 \%$, depending on the water wire configuration. Assuming a Boltzmann distribution, this implies a reduction in proton-hopping free-energy barrier by $\sim 0.125-0.98 \mathrm{~kJ} / \mathrm{mol}$ mediated by NQE. Therefore, although the molecular environment is clearly different in (overall electroneutral) bulk ice VII to explicitly charged water wires, we would expect $\mathrm{NQE}$ to reduce the free-energy barrier for protonic hopping diffusion.

\section{CONCLUSIONS}

We used AIMD, featuring state-of-the-art DRSLL nonlocal dispersion, to study the structural, hydrogen bonding, electronic, vibrational, and Raman properties of ice VII in the pressure range from 5 to $20 \mathrm{GPa}$ to elucidate any possible hints of intramolecular strain that may serve as a precursor for interstitial proton-hopping events to occur. Although we do not detect any water-dissociation and proton-hopping events per se, owing to still-large water-dissociation free-energy barriers, we found reasonable agreement of Raman vibrational band with available experimental data justifying the quality of the applied non-local dispersion treatment and functional. The use of biased-simulation methods, e.g., umbrella sampling to probe the proton-hopping free-energy landscapes, would certainly be important, ideally in conjunction with the path-integral treatment of nuclear coordinates, as would the possibility of external stresses on the system to induce potential hopping.

A formidable challenge for future molecular simulation lies in the accurate determination of NQE-led changes to, and modulation of, the (finite-temperature) proton-hopping freeenergy landscape in condensed phases of water, such as highpressure ice polymorphs.

\section{SUPPLEMENTARY MATERIAL}

See supplementary material for distribution of the $\mathrm{O}-\mathrm{H}$ bond lengths and $\mathrm{H}-\mathrm{O}-\mathrm{H}$ angles, Hirshfeld atomic charges, molecular dipole moments, and further VDOS and IR spectra.

\section{ACKNOWLEDGMENTS}

We thank the Science Foundation Ireland for funding under Grant No. 15/ERC/I3142, as well as John Tse and 
Christian Burnham for interesting discussions, and J.T. for help with initial coordinates.

${ }^{1}$ W. F. Kuhs, J. L. Finney, C. Vettier, and D. V. Bliss, J. Chem. Phys. 81, 3612 (1984).

${ }^{2}$ J. D. Bernal and R. H. Fowler, J. Chem. Phys. 1, 515 (1933).

${ }^{3}$ R. J. Nelmes, J. S. Loveday, W. G. Marshall, G. Hamel, J. M. Besson, and S. Klotz, Phys. Rev. Lett. 81, 2719 (1998).

${ }^{4}$ M. Benoit, D. Marx, and M. Parrinello, Nature 392, 258 (1998).

${ }^{5}$ P. Loubeyre, R. LeToullec, E. Wolanin, M. Hanfland, and D. Husermann, Nature 397, 503 (1999).

${ }^{6}$ M. Somayazulu, J. Shu, C. S. Zha, A. F. Goncharov, O. Tschauner, H. K. Mao, and R. J. Hemley, J. Chem. Phys. 128, 064510 (2008).

${ }^{7}$ H. Hirai, H. Kadobayashi, T. Matsuoka, Y. Ohishi, and Y. Yamamoto, High Pressure Res. 34, 289 (2014).

${ }^{8}$ P. Pruzan, J. C. Chervin, and M. Gaythie, Europhys. Lett. 13, 81 (1990).

${ }^{9}$ P. Pruzan, J. C. Chervin, E. Wolanin, B. Canny, M. Gauthier, and M. Hanfland, J. Raman Spectrosc. 34, 591 (2003).

${ }^{10}$ M. Guthrie, R. Boehler, C. A. Tulk, J. J. Molaison, A. M. dos Santos, K. Li, and R. J. Hemley, Proc. Natl. Acad. Sci. U. S. A. 110, 10552 (2013).

${ }^{11}$ T. Iitaka, H. Fukui, Z. Li, N. Hiraoka, and T. Irifune, Sci. Rep. 5, 12551 (2015).

${ }^{12}$ T. Okada, T. Iitaka, T. Yagi, and K. Aoki, Sci. Rep. 4, 5778 (2014).

${ }^{13}$ E. Katoh, H. Yamawaki, H. Fujihisa, M. Sakashita, and K. Aoki, Science 295, 1264 (2002).

${ }^{14}$ N. Noguchi and T. Okuchi, J. Chem. Phys. 144, 234503 (2016).

${ }^{15}$ M. Dion, H. Rydberg, E. Schröder, D. C. Langreth, and B. I. Lundqvist, Phys. Rev. Lett. 92, 246401 (2004).

${ }^{16}$ G. Román-Pérez and J. M. Soler, Phys. Rev. Lett. 103, 096102 (2009).

${ }^{17}$ J. Wang, G. Román-Pérez, J. M. Soler, E. Artacho, and M.-V. FernándezSerra, J. Chem. Phys. 134, 024516 (2011).

${ }^{18}$ C. Zhang, J. Wu, G. Galli, and F. Gygi, J. Chem. Theory Comput. 7, 3054 (2011).
${ }^{19}$ F. Corsetti, E. Artacho, J. M. Soler, S. S. Alexandre, and M.-V. FernándezSerra, J. Chem. Phys. 139, 194502 (2013).

${ }^{20}$ A. Bankura, A. Karmakar, V. Carnevale, A. Chandra, and M. L. Klein, J. Phys. Chem. C 118, 29401 (2014).

${ }^{21}$ N. J. English, Energies 8, 9383 (2015).

${ }^{22}$ M. J. Gillan, D. Alfè, and A. Michaelides, J. Chem. Phys. 144, 130901 (2016).

${ }^{23}$ Z. Futera and N. J. English, J. Chem. Phys. 147, 031102 (2017).

${ }^{24}$ Z. Futera, M. Celli, L. del Rosso, C. J. Burnham, L. Ulivi, and N. J. English, J. Phys. Chem. C 121, 3690 (2017).

${ }^{25}$ T. D. Kuhne, M. Krack, F. R. Mohamed, and M. Parrinello, Phys. Rev. Lett. 98, 066401 (2007).

${ }^{26}$ T. D. Kuhne, M. Krack, and M. Parrinello, J. Chem. Theory Comput. 5, 235 (2009).

${ }^{27}$ S. Goedecker, M. Teter, and J. Hutter, Phys. Rev. B 54, 1703 (1996).

${ }^{28}$ J. Hutter, M. Iannuzzi, F. Schiffmann, and J. VandeVondele, Wiley Interdiscip. Rev.: Comput. Mol. Sci. 4, 15 (2014).

${ }^{29}$ N. Wiener, Acta Math. 55, 117 (1930).

${ }^{30}$ A. Khintchine, Math. Ann. 109, 604 (1934).

${ }^{31}$ V. F. Petrenko and R. W. Whitworth, Physics of Ice (Oxford University Press, Inc., New York, 1999).

${ }^{32}$ C. J. Burnham and N. J. English, "Free-energy calculations of the intercage hopping barriers of hydrogen molecules in clathrate hydrates," J. Phys. Chem. C 120, 16561-16567 (2016).

${ }^{33}$ C. J. Burnham, Z. Futera, and N. J. English, "Quantum and classical intercage hopping of hydrogen molecules in clathrate hydrate: Temperature and cage-occupation effects," Phys. Chem. Chem. Phys. 19, 717-728 (2017).

${ }^{34}$ C. J. Burnham, Z. Futera, and N. J. English, "Study of hydrogen-molecule guests in type II clathrate hydrates using a force-matched potential model parameterised from $a b$ initio molecular dynamics," J. Chem. Phys. 148, 102323 (2018).

${ }^{35}$ M. Rossi, M. Ceriotti, and D. E. Manolopoulos, "Nuclear quantum effects in $\mathrm{H}+$ and $\mathrm{OH}-$ diffusion along confined water wires," J. Phys. Chem. Lett. 7, 3001-3007 (2016). 